\title{
Una experiencia virtual de aprendizaje sobre la educación estocástica inicial con estudiantes costarricenses de secundaria
}

\author{
A virtual learning experience on initial stochastic education with Costa Rican \\ high school students
}

\section{Uma experiência virtual da aprendizagem sobre a educação estocástica inicial com estudantes de secundária costarriquenhos}

\author{
Rodolfo David Fallas-Soto \\ Universidad de Costa Rica \\ San José, Costa Rica \\ rodolfo.fallas@ucr.ac.cr \\ (1) https://orcid.org/0000-0003-0408-5446
}

Recibido - Received - Recebido: 06 / 03 / 2021 Corregido - Revised - Revisado: 14 / 05 / 2021 Aceptado - Accepted - Aprovado: 25 / 05 / 2021

DOI: https://doi.org/10.22458/ie.v23i34.3452

URL: https://revistas.uned.ac.cr/index.php/innovaciones/article/view/3452

\begin{abstract}
Resumen: Este estudio tiene como objetivo desarrollar, por medio de un entorno virtual, un espacio para la construcción de significados que se asocien con los conceptos básicos de estadística, como parte del proyecto de Comunidades de Aprendizaje Matemático de la Universidad de Costa Rica y los cursos libres que imparte. Para ello, desde el enfoque socio-epistemológico, se desarrollan las etapas de la ingeniería didáctica para implementar en un grupo de 30 estudiantes (entre 14 y 15 años) el ciclo de investigación estadística propuesto por (Wild y Pfannkuch, 1999).

En los resultados se muestran dos organizaciones de prácticas asociadas al actuar del estudiantado cuando comprenden comportamientos de naturaleza estocástica. Además, esta investigación reporta el papel del cambio en la muestra estadística, para asegurar una representatividad de la población de estudio. Se concluye con algunas características de la práctica de inferencia y su relación con una forma de pensar ante comportamientos no deterministas.
\end{abstract}

Palabras clave: Socioepistemología, Aprendizaje en línea, Estadística, Organización de prácticas, Matemáticas, Educación

Summary: This study aims to develop, through a virtual environment, a space for the construction of meanings that are associated with the basic concepts of statistics, as part of the project Mathematical Learning Communities of the University of Costa Rica and the free courses it imparts. For this, from the socio-epistemological approach, the didactic engineering stages are developed to implement in a group of 30 students (between 14 and 15 years old) the statistical research cycle proposed by (Wild and Pfannkuch, 1999). The results show two organizational practices associated with the actions of the students when they understand behaviors of a stochastic nature. In addition, this research reports the role of change in the statistical sample, to ensure a representativeness of the population being studied. It concludes with some characteristics of inference practice and its relationship with a way of thinking about non-deterministic behaviors.

Keywords: Socioepistemology, Online learning, Statistics, Organizational practices, Mathematics, Education

Resumo: Este estudo tem como objetivo desenvolver, por meio de um ambiente virtual, um espaço para a construção dos significados associados aos conceitos básicos da estatística como parte do projeto de Comunidades de Aprendizagem Matemática da Universidade da Costa Rica e dos cursos livres que ali são ministrados. Para isso, desde uma abordagem sócio-epistemológica foram desenvolvidas etapas de engenharia didática para serem executadas em um grupo de 30 estudantes (entre 14 e 15 anos) no ciclo de pesquisa estatística proposto (Wild e Pfannkuch, 1999). Nos resultados serão mostradas duas organizações de práticas associadas ao agir do corpo estudantil quando compreendem comportamentos de natureza estocástica. Além do mais, esta pesquisa mostra o papel da mudança estatística para garantir a representatividade da população em estudo. Concluiu-se que algumas características da prática de inferência e sua relação com uma forma de pensar diante de comportamentos não deterministas.

Palavras chave: Socioepistemologia, aprendizagem online, estatística, organização de práticas, matemática, educação 


\section{INTRODUCCIÓN}

La educación estocástica promueve un espacio para el aprendizaje y la enseñanza de la estadística combinatoria y probabilística de manera articulada, esto posibilita el desarrollo de una forma particular de pensamiento vinculado con el estudio de fenómenos aleatorios, interpretación de muestras y construcción de inferencias (Lopes, 2012).

Este trabajo tiene por objetivo desarrollar un espacio -por medio de un entorno virtual-, en el cual un grupo de estudiantes de tercer año de secundaria en Costa Rica construya un significado para las nociones estadísticas básicas propuestas en el plan de estudios del Ministerio de Educación Pública (2012).

Durante el 2020 se disemina la pandemia por el virus responsable de la COVID-19 (Peralta, Carozzo, Sierra y Bu, 2020), una de las consecuencias de este hecho en la educación costarricense fue la virtualización y la enseñanza a distancia para los diferentes niveles educativos en el país; en ese sentido, "el sistema educativo dio un giro rotundo de la presencialidad tradicional a la remota" (Carranza-Marchena y Zamora-Sánchez, 2020). En algunos casos, lo anterior provocó explorar los entornos virtuales de aprendizaje e indagar sobre metodologías para que el estudiantado viviera procesos de aprendizaje desde sus hogares con apoyo de internet.

El Ministerio de Salud en Costa Rica promovió diferentes medidas sanitarias como el aislamiento social ante la pandemia (Vega-Jiménez, 2021). Particularmente, esto representó un reto para el proyecto llamado Comunidades de Aprendizaje Matemático (ED3569) que desarrolla el Departamento de Educación Matemática de la Escuela de Matemática de la Universidad de Costa Rica; cuyo objetivo es propiciar el mejoramiento de la formación matemática en la educación pública primaria y secundaria, mediante la oferta de cursos y talleres presenciales, virtuales o bimodales de corto y mediano plazo, en diversidad de temas matemáticos y didáctico-matemáticos, dirigidos a estudiantes, docentes o familiares responsables del estudiantado. A partir de la pandemia, el proyecto sigue en pie, pero ahora desde la modalidad virtual.

Cabe resaltar que desde hace unos ocho años el Departamento de Educación Matemática ha venido trabajando con el fin de que el profesorado incluya en sus cursos el uso de la tecnología, tanto en los procesos de enseñanza como de aprendizaje, de modo que los estudiantes y los docentes han ido desarrollando una familiarización con las herramientas y su uso en el aula de matemática.

Lo anterior descrito, generó que la mayoría de las personas facilitadoras de llevar a cabo talleres al personal docente o clases al estudiantado dentro del proyecto, se encontraran preparadas ante la necesidad de incorporar lo virtual en los procesos de enseñanza y aprendizaje, lo cual permitió crear una pronta respuesta de apoyo: así nace la propuesta de Comunidades Virtuales de Aprendizaje Matemático.

Ese colectivo de facilitadores forman parte del colectivo docente o estudiantil de la Escuela de Matemática quienes, de manera voluntaria, donan su tiempo y sus recursos en la realización de planeamiento y las reuniones de coordinación, tanto para las sesiones sincrónicas como las asincrónicas.

Aunado a lo anterior, el contexto actual de la emergencia sanitaria viene a contribuir de manera importante a la interrupción parcial o total de las clases presenciales en el país, por una diversidad de factores socio-económicos de la región. Desde esta problemática, este proyecto de acción social busca evitar la exclusión de estudiantes del sistema educativo y velar por la democratización del aprendizaje matemático para la población estudiantil costarricense.

A su vez, este nuevo panorama, ha permitido concebir el proyecto original en dimensiones mayores, porque la virtualidad de los procesos educativos y acompañamiento docente resulta un poderoso mecanismo para acceder a poblaciones lejanas y más vulnerables, las cuales las propuestas en modalidad presencial tradicionalmente dejan por fuera o atienden con menor frecuencia. 
Lo anterior no significa que se ignoran las dificultades de acceso a recursos tecnológicos y al internet de muchas personas de distintas zonas del país, pero sí se concibe la apertura de muchos actores sociales hacia la importancia y la necesidad de la creación de espacios y recursos para concebir la virtualidad como una oportunidad de acceso hacia más y mejores procesos educativos.

En este proyecto se trabaja actualmente en una modalidad de educación virtual, caracterizada por utilizar el internet mediante entornos virtuales como el medio principal para desarrollar espacios para vivir los procesos de enseñanza y aprendizaje (Barboza Robles, 2020, p. 90).

Asimismo, se anuncia la apertura de talleres o cursos libres por medio de redes sociales de la Universidad de Costa Rica o notas periodísticas para que familiares responsables del estudiantado menor de edad realicen la matrícula. Desde la descripción de este escenario, se desarrolla particularmente una propuesta didáctica para la construcción de conocimiento estadístico a 30 estudiantes de tercer año de secundaria de centros educativos públicos (entre 14 y 15 años).

Una de las propuestas para la enseñanza y el aprendizaje de la estadística es utilizar ciclos de investigación, para que los estudiantes puedan utilizar los conocimientos adquiridos en las lecciones; sin embargo, a pesar de utilizar problemas en contextos situacionales reales, estos podrían carecer de funcionalidad para la vida del estudiante y su contexto.

No obstante, la propuesta que se presenta efectúa lo contrario y se enfoca en una problemática dentro del contexto real de la población estudiantil, que además sea funcional; $y$, a partir de ahí, puedan buscar y aprender herramientas para el análisis y la comunicación de los datos, con la construcción de conocimiento estadístico a partir su uso. Al ser esto parte de la educación estocástica, se busca que esa construcción de conocimiento articule nociones de la estadística descriptiva e inferencial, en el estudio de la información y la construcción de conclusiones e inferencias realizadas a una población definida por el proyecto que desarrolla el estudiantado.

En la experiencia didáctica que se presenta en este artículo, el estudiantado propone y desarrolla un proyecto para determinar las actividades que realiza la población en Costa Rica, con el fin de sobrellevar el cambio de vida a consecuencia de la pandemia.

Cada integrante de la clase pertenece a diferentes localidades del país, esto contribuyó a recolectar información desde diferentes puntos geográficos, con apoyo de una encuesta en línea y redes sociales. En consecuencia, cada día se evidencia un aumento en los datos por la cantidad de respuestas obtenidas.

\section{Fundamentación teórica}

Esta experiencia didáctica se sustenta desde la Teoría Socio-epistemológica de la Matemática Educativa. En ese sentido, Cantoral (2019) señala que para resolver problemáticas o desarrollar propuestas de situaciones de aprendizaje desde este enfoque teórico, se requiere de una doble descentración: descentración del objeto y de sus modelos didácticos para poner la atención en las prácticas, las cuales ayudan a la construcción del conocimiento.

En esta Teoría se desarrolla el Pensamiento y Lenguaje Variacional como línea de investigación, la cual propone estudiar la variación de forma más integral en escenarios de construcción del conocimiento, no solamente asociado al cálculo o al análisis matemático; sino como una forma de pensamiento que está en la base del pensamiento humano que puede iniciar con la comparación de estados como una acción de la persona. 
Si se considera esta hipótesis y específicamente en esta propuesta, se cree que, al desarrollar el ciclo de investigación estadística con apoyo de recursos en línea, se presente una forma particular de estudiar el cambio que ayuda a la construcción de datos e inferencias. Por la naturaleza de comportamientos azarosos, no podemos dar predicciones sobre una población de estudio, pero si inferencias; y es que -el aumento en la cantidad de las respuestas en la recolección de datos-, día con día, permite al estudiante construir y modificar inferencias o conjeturas sobre la población en estudio, así como la construcción del dato.

La encuesta que diseñó el estudiantado se mantuvo dos semanas en línea, esto permitió construir inferencias en los primeros seis días con los datos proporcionados de 396 personas. En la segunda semana, se tienen 534 respuestas, ante esto, ¿qué tanto cambiaron las inferencias o conclusiones que se tenían?, y ¿qué nuevas inferencias podría construir ahora el estudiantado?

Ante ese panorama se reconoce un cambio en la cantidad de respuestas de una encuesta en línea ante un comportamiento no determinista de los datos; pues no se puede predecir el comportamiento de toda una población con esta muestra. El reconocer este cambio permite identificar y explicar con herramientas estocásticas al relativismo de las inferencias o conclusiones sobre la población ante el tamaño de la muestra.

Lo anteriormente mencionado, articula elementos de la estadística descriptiva con la estadística inferencial, herramientas que permiten al estudiante dar inferencias sobre una población a partir de una muestra (quienes responden la encuesta) y familiarizarse con la naturaleza estocástica de estos fenómenos (no determinista).

Con respecto a la Socioepistemología, reconoce e integra dos tipos de constructivismo: el cognitivo (Piaget, 1976) basado en los principios de cognición como acciones interiorizadas de la persona expuestas ante una situación y, por otro lado, el social (Vygotsky, 1986) como actividades internalizadas como coordinación racional de acciones del individuo ante una situación en colectivo y en contexto.

Por lo anterior, esta Teoría considera estos elementos organizados por prácticas socialmente compartidas en la comprensión de usos y significados compartidos desde el individuo al colectivo, modelo que se presenta en la siguiente figura (figura 1). Esto es, centrarnos en una organización de prácticas por sobre una evolución de conceptos.

Figura 1. Parte del modelo de anidación de prácticas de la Teoría Socio-epistemológica de la Matemática Educativa

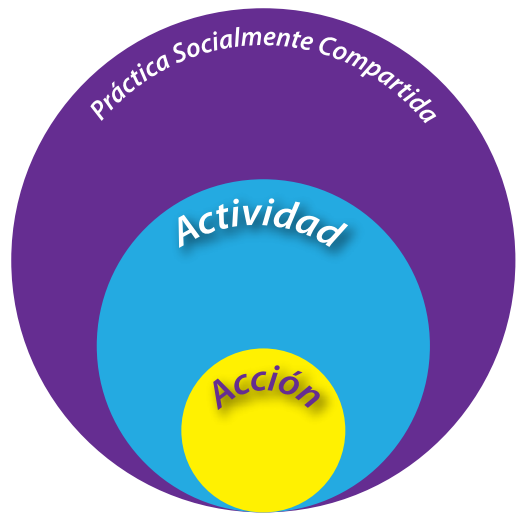

Fuente: elaboración propia, basado en el modelo de Cantoral (2019, p. 3). 
Como se muestra en el modelo, las prácticas o prácticas socialmente compartidas permiten lograr el objetivo o meta de un grupo o individuo frente a un problema o situación, normada y continuada a nivel social. Por otro lado, las acciones son el primer acercamiento que tiene la persona o grupo cultural frente a una situación, tal que un conjunto de esas acciones organizadas de manera sistemática forma actividades que dan lugar a obtener el objetivo del grupo cultural o individual; de tal manera, se consolida como una práctica socialmente compartida.

Por ejemplo, más adelante en los resultados de esta investigación se determina una organización de prácticas: comparación de los datos (acción) para la construcción de un dato (actividad, como explicación racional de la persona al grupo cultural sobre lo que considera por dato), para conjeturar con respecto a la problemática abordada (en colectivo se crean conjeturas de lo que el dato les ofrece). Esta anidación permitió al estudiante construir conocimiento estadístico.

La Socioepistemología descansa en cuatro principios que actúan siempre de forma articulada en los trabajos que se abordan desde este enfoque (Cantoral, Montiel y Reyes-Gasperini, 2015), estos son:

1. Racionalidad contextualizada: alude en reconocer, dar lugar y evidenciar la racionalidad de cada uno de los entes relacionados con la construcción de conocimiento en un cierto contexto y realidad (comunidades, personas, libros, obras, producciones, entre otros).

2. Relativismo epistemológico: reconocer que existen diversas formas de construir, dar significado y usar al conocimiento; es dar relatividad a lo que comprendemos por saber.

3. Resignificación progresiva: abarca más allá de solamente dar diferentes significados a un objeto. Es enriquecer al conocimiento reconociendo una evolución y ramificación en su significado desde su construcción y uso en diferentes quehaceres, contextos, realidades y momentos en la vida.

4. Normatividad de la práctica social: es el principio que permite lograr la significación de la matemática mediante el uso, plasmado en una organización de prácticas socialmente compartidas (la triada acciones, actividades y prácticas), normadas por la práctica social (lo que nos hace hacer lo que hacemos).

Parte de los objetivos de trabajos enmarcados en esta Teoría es proponer y estudiar una forma de intervención dentro o fuera de la escuela para la construcción de conocimiento: situación de aprendizaje. Una situación de aprendizaje es un conjunto de momentos en un escenario real o hipotético, en el cual el individuo inhibe el uso exclusivo de recursos mnemotécnicos o técnicas, para empezar a cuestionar, razonar, argumentar; a tal punto que hace más evidente el uso de prácticas.

En síntesis, lo que se observa de su implementación es el actuar de una persona o colectivo en la construcción de conocimiento, principalmente se valida a una organización de prácticas que transforman la manera tradicional en que se organiza el currículum, por una organización de contenidos, esto es, descentrarse del objeto.

Dentro de la educación estocástica se desarrollan estrategias como ciclos de investigación estadística, los cuales invitan al estudiantado a utilizar herramientas estadísticas o probabilísticas para el análisis de los datos, siendo de utilidad en la vida y para la toma de decisiones de quien aprende. Lo anterior indica que las personas en este escenario estarán en modo situación de aprendizaje, caracterizada desde la Socioepistemología; el reto de este trabajo es identificar y validar organizaciones de prácticas en el actuar del estudiantado. 


\section{MATERIALES Y MÉTODOS}

Esta investigación tiene un enfoque cualitativo, con el cual se analiza el actuar del estudiantado en la construcción colectiva de su proyecto de investigación estadística. Para lograrlo se recolecta información con apoyo de grabaciones de las clases, por medio de una plataforma de video y audio, conferencia en línea como instrumento de la técnica de observación.

En cuanto a las etapas de la Ingeniería Didáctica, Artigue (2000) indica que se conforma de las siguientes etapas: análisis preliminar, análisis a priori, implementación del diseño y análisis a posteriori. Cada una se describe a continuación.

1. En el análisis preliminar se desarrolla una problematización del saber matemático que corresponde a un método utilizado por las investigaciones de corte Socio-epistemológico, que tiene muchas rutas metodológicas y ayuda a cambiar la relación con el saber; es decir, dejar de centrarnos en una regresión o evolución conceptual (temas o contenidos) a centrarnos en las prácticas que acompañan a la construcción de objetos matemáticos. Este método consiste en estudiar un conocimiento matemático en uso desde uno o varios escenarios de un saber técnico, popular o científico; situado contextual y temporalmente, el cual ayuda a confrontar los conocimientos institucionalizados en la matemática escolar. Dos mecanismos que caracterizan a este método es historizar y dialectizar.

Para este caso, la historización consiste en reconocer el valor histórico de trabajos desarrollados alrededor de la educación estocástica y el diálogo con colegas que investigan en esta línea, conectando con una epistemología situada y una historia social del objeto. Vásquez Ortiz (2020) brinda reflexiones sobre el papel de la educación estocástica en la formación de ciudadanos para el desarrollo sostenible:

En este sentido, es imperativo incorporar la educación del desarrollo sostenible en la práctica educativa, lo que implica una manera diferente de trabajar en el aula, a través de una enseñanza conectada con el contexto y que aborde problemáticas actuales, que impulse un pensamiento crítico, que permita a los estudiantes comprender la realidad. Sobre todo, considerando que el principal objetivo de la EDS es educar para la acción (párr.14).

Vásquez Ortiz invita a reflexionar sobre la importancia de considerar un contexto situacional real; pero además funcional para la vida del estudiante y su entorno, que los guíen a desarrollar pensamiento estocástico ante los desafíos más apremiantes del mundo actual.

Con esta visión (Alsina, Vásquez, Muñiz-Rodríguez, y Rodríguez-Muñiz, 2020) dan propuestas y estrategias para promover la alfabetización estadística y probabilística en contexto para educación primaria.

Con esto, se inicia una confrontación del conocimiento matemático escolar institucionalizado, esto es la dialectización, mecanismo que invita a una confrontación necesaria para la comprensión de otros significados y racionalidades, en aquello que se asume como un conocimiento construido y podría gozar de veracidad absoluta en el escenario escolar o institucionalizado en los libros de texto.

Estos mecanismos ayudan a la reflexión del saber matemático en las dimensiones: didáctica, cognitiva, epistemológica y social; está última permea, modifica, integra y sistematiza las demás dimensiones. Tanto en Vásquez Ortiz (2020) como en Alsina et al. (2020) retoman el ciclo de investigación estadística propuesto por Wild y Pfannkuch (1999) (Figura 2). 
Figura 2. Ciclo de investigación estadística.

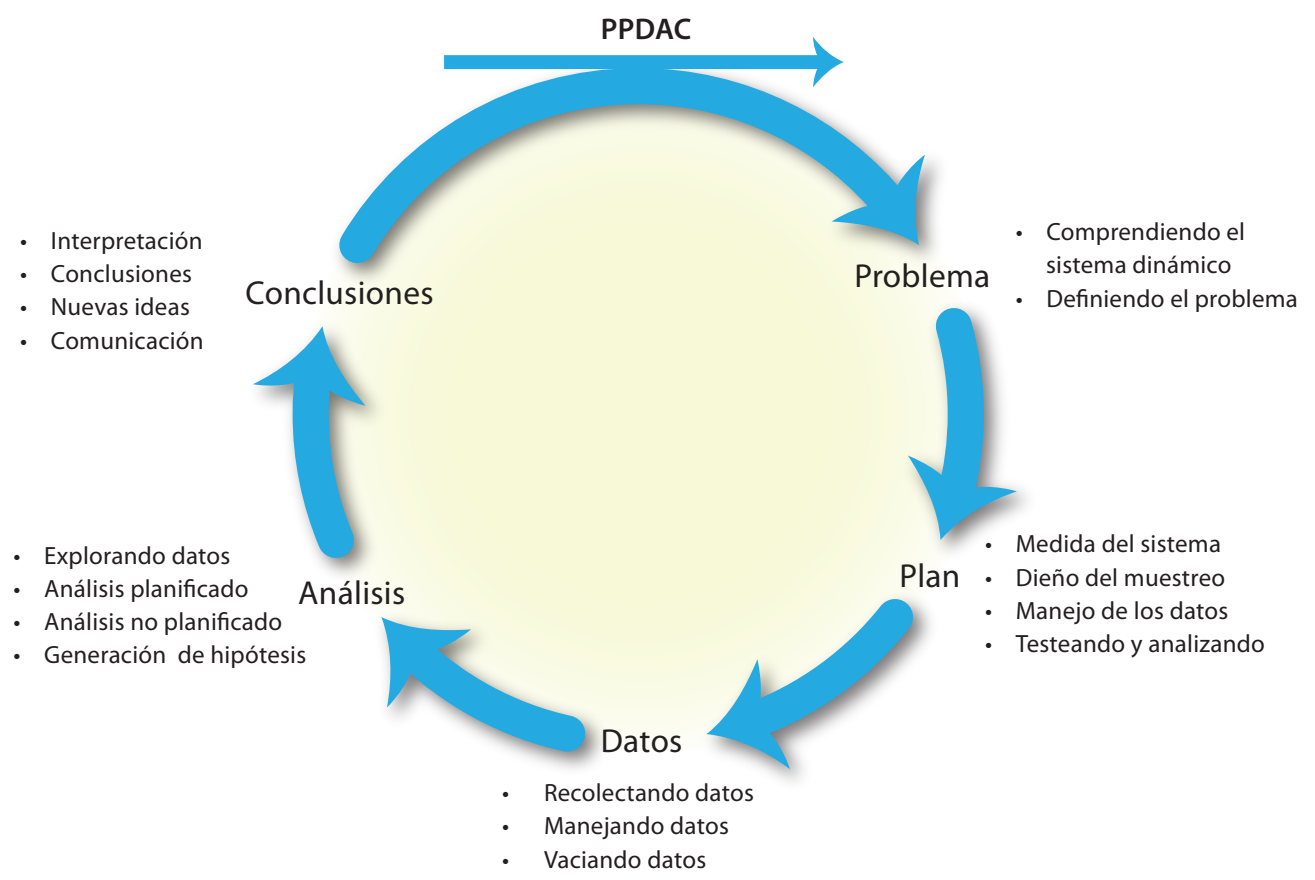

Fuente: tomado de Vásquez Ortiz (2020, p. 14) que traduce de (Wild y Pfannkuch, 1999).

2. En el análisis a priori se estudia este ciclo conocido como PPDAC por sus siglas, las cuales significan Problema-Plan-Datos-Análisis-Conclusiones, se convierte en la ruta para guiar la propuesta del proyecto en estadística que construye el estudiantado. En este momento no se tienen prácticas asociadas, pero al llevar a cabo este ciclo dará evidencia de las prácticas que ayudan a construir objetos estadísticos.

En esta experiencia de aprendizaje se trabaja con 30 estudiantes de tercer año de secundaria de instituciones públicas del país. A estos estudiantes los matricularon sus responsables de familia, quienes vieron anuncios o noticias sobre los cursos libres que ofrece el proyecto de acción social de Comunidades de Aprendizaje Matemático del Departamento de Educación Matemática de la Universidad de Costa Rica.

La muestra estudiantil pertenece a las siguientes instituciones de educación secundaria pública: IEGB América Central, CTP Pacayas, Liceo de Aserrí, Liceo José Joaquín Jiménez Núñez, Liceo Rodrigo Facio Brenes, Conservatorio de Castella, CTP San Isidro de Pérez Zeledón, Liceo San Antonio, CTP José María Zeledón Brenes, Liceo Laguna, Liceo Vuelta de Jorco, Liceo de Calle Fallas, CTP Liverpool y CTP Corredores.

3. En la etapa de implementación del diseño, se desarrolla el ciclo PPDAC en seis sesiones de dos horas con apoyo de la plataforma Zoom ${ }^{\circledR}$ y de herramientas digitales como buscadores web, Google Forms ${ }^{\circledast}$ y MS Excel ${ }^{\circ}$.

4. Para el análisis a posteriori consiste en el estudio del actuar de la población estudiantil ante el ciclo PPDAC, se utiliza un instrumento para la recolección de los datos, ahí se consideran dos categorías de análisis con su respectivo indicador y dato, a partir de grabaciones obtenidas del actuar de los estudiantes: 
a) La primera categoría de análisis corresponde al "rol del cambio en la muestra para la construcción de inferencias sobre la población", provocado por la naturaleza de la encuesta al estar en línea y donde cada día aumenta la muestra y se obtienen nuevas respuestas. Los indicadores corresponden a estudiar cómo los estudiantes reconocen este cambio, el cómo y cuánto afecta en el análisis; de tal manera que se reconoce la naturaleza estocástica de la problemática.

b) La segunda categoría de análisis llamada "desarrollo intencional de prácticas", se identifican y validan con los argumentos del estudiantado a las acciones, actividades y prácticas, de tal forma que construyen una organización de prácticas. Los indicadores giran entorno de lo que se observa en el hacer de las personas: ¿qué hace?, ¿cómo lo hace? y ¿para qué lo hace?, propuesto por (Cantoral, Montiel, y Reyes-Gasperini, 2015a). Esto ayuda a determinar las prácticas asociadas a la construcción de conocimiento y significados asociados del objeto estadístico.

TABLA 1

Categorías de análisis, indicadores y datos para el análisis del hacer de los estudiantes al desarrollar el ciclo PPDAC

Categoría de análisis

Rol del cambio en la muestra para la construcción de inferencias sobre la población

Desarrollo intencional de prácticas
Indicadores

¿Qué cambia?

¿Cómo afecta ese cambio?

¿Cuánto afecta ese cambio?

¿Qué hace?

¿Cómo lo hace?

¿Para qué lo hace?
Dato

Construcción de conjeturas

Características de naturaleza estocásticas

Prácticas y significado del objeto

Fuente: elaboración propia.

\section{DISCUSIÓN DE RESULTADOS}

En esta sección se presentan dos clases de resultados: los obtenidos por el grupo de estudiantes sobre el proyecto construido denominado: "Salud y Pandemia" y el docente-investigador al desarrollar el ciclo PPDAC, ante una problemática que construyeron; y lo analizado por el docente-investigador en el hacer de los estudiantes.

El hallazgo principal fue determinar una evolución pragmática (organización de prácticas) involucrada en la construcción de conceptos no tan típicamente escolares; pues algunos eran contenidos que aparecen en los programas de estudios (Ministerio de Educación Pública, 2012) y otros no, pero que jugaron un papel funcional durante el desarrollo del ciclo PPDAC.

\section{Ciclo de investigación estadística: salud y pandemia}

Para la escogencia de la problemática a desarrollar se creó una lista con las ideas que brindan los estudiantes, agrupándolos por temáticas (Figura 3). Sobre esto, los temas en en los cuales presentaron interés son: cuestiones económicas de estudiantes costarricenses, la educación en Costa Rica, la alimentación y la influencia de las grandes empresas transnacionales, acciones ante la contaminación ambiental, la salud y la pandemia, abusos o violencia (xenofobia, racismo, género, etc.) y la justicia en el país.

Un criterio del grupo estudiantil para escoger el tema es lograr comunicar los resultados que obtuvieran para informar y ayudar a la población dentro y fuera de Costa Rica durante la crisis provocada por la pandemia. Por lo tanto, la problemática abordada lleva por nombre: Salud y Pandemia. 
Figura 3. Ideas de los estudiantes para el planteamiento del problema.

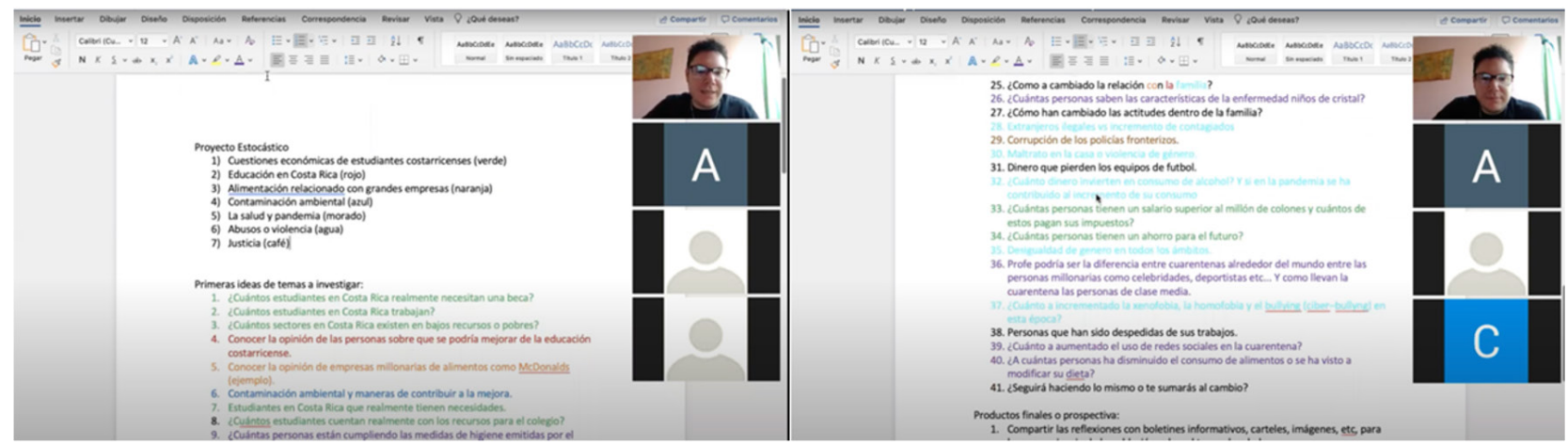

Fuente: elaboración propia.

Para trabajar en un plan de desarrollo, toman la decisión de buscar y estudiar fuentes para defender el problema. Los estudiantes reflexionaron sobre documentos que ellos mismos encontraron, por mencionar algunos: Huarcaya-Victoria (2020); Nieuwenhuijsen (9 de abril de 2020); Ornell, Schuch, Sordi y Kessler (2020) y Ramírez-Ortiz, Castro-Quintero, Lerma-Córdoba, Yela-Ceballos, y Escobar-Córdoba (2020).

Las discusiones iniciales en la clase virtual evidenciaban el foco hacía consecuencias de la salud por contagio; pero al compartir los resultados y las conclusiones de estos artículos, provocaron nuevas reflexiones que orientaron la mirada a las consecuencias psicosociales que provocan estos escenarios de pandemia; como: estrés, depresión, ansiedad, insomnio, deterioro en la organización individual y relaciones sociales, entre otras. Dentro de las recomendaciones que dan los autores que coinciden con los resultados obtenidos por el colectivo estudiantil, es continuar en lo posible con las rutinas habituales; para ello se deben tener en cuenta las medidas preventivas, mantenerse ocupado, distribuir el tiempo de las necesidades básicas, evitar drogas y alcohol, pasar tiempo con la familia, no saturarse de información con temas de la pandemia, expresar sus sentimientos a un profesional psicológico o personas de confianza, entre otras.

En consecuencia, se decide tomar datos que ayuden a responder el problema del estudio construido: ¿cómo se ve afectada la salud (mental y física) de la persona en esta época de aislamiento provocado por la pandemia? Con este problema se determinó lo que ha cambiado en la vida de las personas durante la pandemia y se obtienen reflexiones para una etapa post-pandemia. Se plantearon preguntas asociadas a la problemática que guiaron a la construcción de la encuesta en línea:

1. ¿Cuántas personas han entrado en estado de negatividad o depresión?

2. ¿Cuántas han aprovechado este tiempo para encontrarse a sí mismos?

También se consideraron sub-temáticas como: la contaminación, la violencia, las consecuencias económicas, políticas, entre otras que podrían estar asociados a la problemática.

Se diseñaron preguntas para la confección de una encuesta en Google Forms, organizada en tres secciones: datos personales, su salud en la cuarentena y reflexiones finales. A continuación, se presenta una tabla sobre las preguntas diseñadas después de tres sesiones para la construcción y la reflexión generada por el colectivo estudiantil: 
TABLA 2

Construcción de la encuesta en línea y reflexiones generadas

1. ¿Cuál es su nacionalidad?

Pregunta diseñada con respuesta abierta; es decir, no tiene opciones como respuesta, variable cualitativa nominal (sustantivos).

2. ¿Vive usted en Costa Rica? Variable cualitativa binaria.

3. ¿Cuál es su edad?

Pregunta diseñada con respuesta cerrada por rango de edades, ordenadas.

4. Personas con las que usted convive dentro y fuera de casa regularmente (oficina, transporte público, etc.)

Pregunta diseñada con respuesta abierta, variable cuantitativa discreta.

5. ¿Cuáles suministros de precaución o cuidados básicos ha utilizado en esta cuarentena?

Pregunta diseñada con respuesta múltiple, variable cualitativa nominal organizada por uso personal, en hogar o espacios exteriores.

6. Con respecto a los gastos del hogar, ¿cuánto dinero ha invertido mensualmente para la compra de suministros de precaución?

7. ¿Siente que ha sido satisfactoria esta inversión?

Pregunta diseñada con respuesta cerrada.

8. ¿Se siente seguro al usar estos suministros para su protección personal?

Pregunta diseñada con respuesta cerrada, variable cualitativa binaria.

Pregunta diseñada con respuesta cerrada, variable cualitativa binaria.

9. ¿Cómo se ha sentido durante este aislamiento?

Pregunta con opción múltiple, variable cualitativa nominal en combinación de 2 entre tenso, cansado, activo, calmado.

10. ¿Cuántas horas en promedio duerme diariamente?

Pregunta diseñada con respuesta cerrada por rango de horas, ordenadas.

11. ¿Cómo calificaría su sensación de descanso al despertar?

Pregunta diseñada con respuesta cerrada, variable cualitativa ordinal.

12. ¿Qué actividades NUEVAS ha hecho en esta cuarentena?

13. ¿Cuántos días a la semana hace ejercicio

Pregunta diseñada con opción múltiple, variable cualitativa nominal.

Variable cuantitativa discreta, pregunta con respuesta abierta.

14. ¿Cuánto tiempo le dedica en promedio por cada día de ejercicio?

Pregunta con respuesta abierta, variable cuantitativa continua, hasta los minutos entran en la respuesta.

15. Si está estudiando o trabajando y la nueva dinámica le ha generado estrés, ¿cómo ha superado dicho estrés?

16. Si es estudiante y después de pasar la pandemia, ¿qué reflexión o consejo le daría a sus profesores sobre el tema de educación?

17. ¿Qué consejos nos podría dar para vivir en esta nueva normalidad?

18. ¿Con qué acciones ha contribuido para cuidar el bienestar de otras personas?

19. ¿Qué cree que mejorará en la sociedad después del Covid-19?

Fuente: elaboración propia.

Estas preguntas las construyen pensando en sus realidades y problemáticas. Quieren contrastar con las respuestas de las encuestas. Además, al ser de desarrollo brindan ideas para responder a la problemática planteada.

Esta encuesta se compartió en las redes sociales de cada uno de los estudiantes y se obtuvo la respuesta de 534 personas nacionales y extranjeros. La información recolectada fue estudiada y colocada en un informe entre el colectivo. De acuerdo con lo anterior, los principales hallazgos y los resultados de los estudiantes fueron presentados en el Seminario de Educación Matemática (Sedumat) que organiza el Departamento de Educación Matemática de la Universidad de Costa Rica. Esto se puede apreciar en el siguiente enlace: https://youtu.be/BlhtUiyiRqU 


\section{El rol del cambio en la muestra para la construcción de inferencias sobre la población}

Con respecto a los resultados de esta investigación sobre esta experiencia didáctica, el papel del cambio en el crecimiento de las respuestas de la encuesta, día con día, jugó un papel importante en la construcción y el análisis de la información. La encuesta tardó doce días circulando en las redes sociales. En ese lapso se observó que a los seis días se tenían 396 respuestas. Esta observación ayudó a que los estudiantes dieran inferencias para poblaciones mayores, como 500 encuestados, o con 20 mil personas o cinco millones (población total aproximada del país), como se observa en el siguiente Episodio NicoTai (figura 4).

Figura 4. Episodio Nico-Tai sobre la representatividad de la muestra y el reconocimiento de comportamientos estocásticos

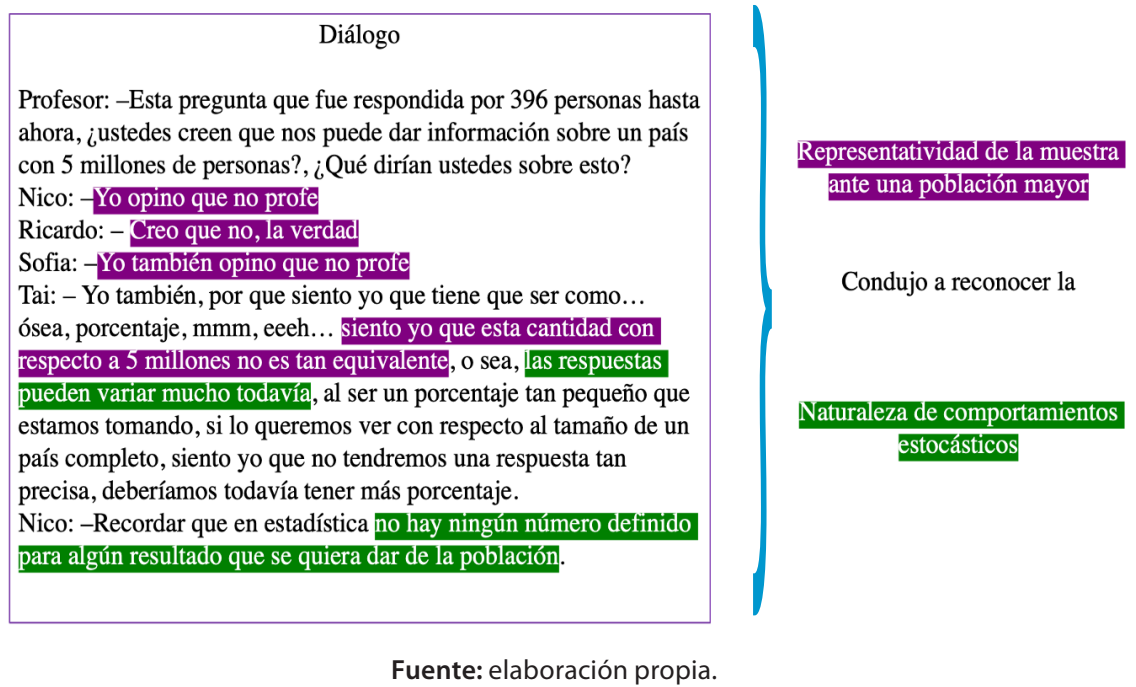

Si bien es conveniente observar cómo el estudiantado discute sobre el cambio asociado al crecimiento de la muestra, se presta atención a la representatividad de la muestra y de este tipo de muestreo para que se pueda utilizar, con el fin de efectuar inferencias sobre la población de un país. Se reflexiona que la inferencia se basa en el muestreo aleatorio y, por lo tanto, el rol del profesor es reflexionar con el estudiantado si el hecho de tener una muestra más grande asegura la representatividad.

Esto aporta a reconocer características de problemas estocásticos, los cuales se tratan poco en el escenario escolar, y son estos los procesos, los modelos o los fenómenos en los cuales existe una secuencia cambiante aleatoriamente de valores que deben ser analizados con herramientas estadísticas y probabilísticas.

Esta percepción del cambio evidencia una integración entre la estadística descriptiva y la inferencial, en la construcción de inferencias o conjeturas a la población sobre el problema de estudio. En esta etapa el colectivo deja de generar suposiciones sobre la población y justifica el grado de incertidumbre que tienen este tipo de problemas y que se deben estudiar estrategias para la representatividad de la muestra y construir inferencias con argumentos. 


\section{Desarrollo intencional de prácticas}

Otro de los resultados, es que al desarrollar el ciclo PPDAC efectivamente propicia la implementación de prácticas en el actuar de los estudiantes en un escenario dentro de un entorno virtual; pues el ciclo no espera como respuesta el buen uso de una fórmula o la buena clasificación de variables, sino que se usan y construyen objetos para analizar la información y tomar decisiones al respecto.

A nivel de acción, el colectivo compara los datos obtenidos en cada pregunta con el propósito de comprender lo que se tiene, sin identificar aún que son datos; pues observan números, frases u opiniones. Esta comparación provoca comunicar en el colectivo lo que se considera por dato en cada una de las preguntas analizadas (construcción del dato como actividad), para luego empezar a realizar conjeturas sobre los datos obtenidos. En la figura 5, se presenta el episodio de Ricardo, quien con su actuar se evidencia esta organización de prácticas.

Figura 5. Episodio Ricardo sobre la organización de prácticas en la organización de los datos

\begin{tabular}{|c|c|}
\hline $\begin{array}{l}\text { Diálogo } \\
\text { Profesor: -Bueno Ricardo, tu pregunta para analizar fue la cantidad } \\
\text { de tiempo invertido por las personas para hacer ejercicio, ¿cómo te } \\
\text { fue? } \\
\text { Ricardo: -Bueno profe, ¿puedo compartir pantalla? } \\
\text { Profesor: -Si claro } \\
\text { Ricardo: -Esteee, bueno, lo que yo hice fue ver un montón de } \\
\text { números que no tenía idea de que hacer con ellos, así que los } \\
\text { ordené. Iba contando, para que al final colocar en una hoja de papel } \\
\text { cuantos ceros, unos, dos, había. Llegaba aquí, por ejemplo, e iba } \\
\text { contando cuantos hacían una hora de ejercicio, cuantos dos, y } \\
\text { revisando que lo que iba contando estaba en línea con la tabla. Ya } \\
\text { los datos, sumados y todo iba poniendo ordenado en cada minuto } \\
\text { las personas que hacian ese tiempo. Y bueno, jaja por acá una } \\
\text { tontera, hubo una persona que respondió que hacía } 32 \text { horas ar día } \\
\text { ejercicio, entonces lo puse como que no lo contestaron y otros } \\
\text { datos que puse como no proporcionados o no importantes fueron } \\
\text { respuestas como que ponian "camino bastante". Esto no seria un } \\
\text { dato para mí, necesitaba números, así que no podría saber con esto } \\
\text { cuantas horas o minutos. Y luego resumí todo esto aún más } \\
\text { haciendo categorías: } 0 \text { a } 1 \text { hora, más de } 1 \text { hora a } 2 \text { horas, más de } 2 \\
\text { horas a } 3 \text { horas, etc. Esto me muestra que un atto porcentaje hace } \\
\text { ejercicio, pero aproximadamente } 131 \text { personas hacen menos de } 30 \\
\text { min o nada de ejercicio para el total de } 519 \text { que respondieron, es un } \\
\text { porcentaje muy alto y preocupa. }\end{array}$ & $\begin{array}{l}\text { Comparación de los datos } \\
\text { (acción) } \\
\text { que lleva a la } \\
\text { Construcción del dato } \\
\text { (actividad), }\end{array}$ \\
\hline
\end{tabular}

Fuente: construcción propia.

Esta organización de prácticas en la recolección de los datos realizada (figura 6) ayudó a construir objetos como: tipos de estadística (descriptiva e inferencial), tipos de preguntas y respuestas (abiertas, cerradas), tipos de variables y su clasificación, sesgos, diferencia entre cuestionario y encuesta, relación entre muestra y población en cada pregunta. También se comentó sobre formas de redactar, información sobre normas o sugerencias de la Caja Costarricense del Seguro Social, entre otros.

Figura 6. Organización de prácticas durante la recolección de datos

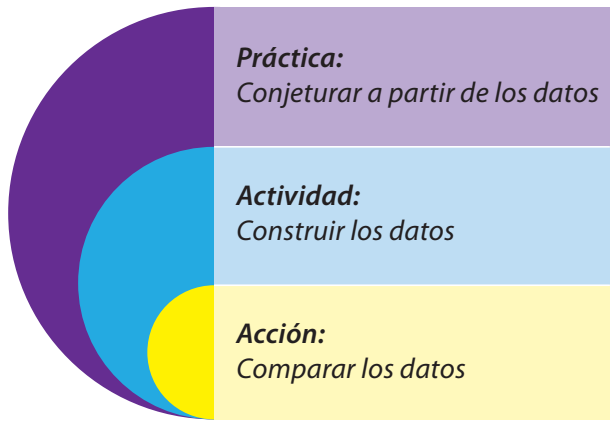

Fuente: elaboración propia. 
Durante la exploración de los datos, la interpretación y la presentación la comunidad estudiantil evidencia otra organización de prácticas, con el objetivo de comunicar los resultados de una forma práctica y clara a partir de un procesamiento de datos para la obtención de la información.

Por ese motivo, en esta etapa, cada estudiante parte del hecho de organizar los datos, darle un orden (acción interiorizada obtenido de la organización de prácticas previa); para proceder a comparar la información que permite formar categorías (actividad) y así visualizarla con apoyo de registros numéricos o gráficos para comunicar más fácilmente la información (práctica). En la figura 7 se presenta el episodio de Alexandra en el cual se evidencia esta organización de prácticas.

Figura 7. Episodio Alexandra sobre la organización de prácticas durante la exploración e interpretación de los datos

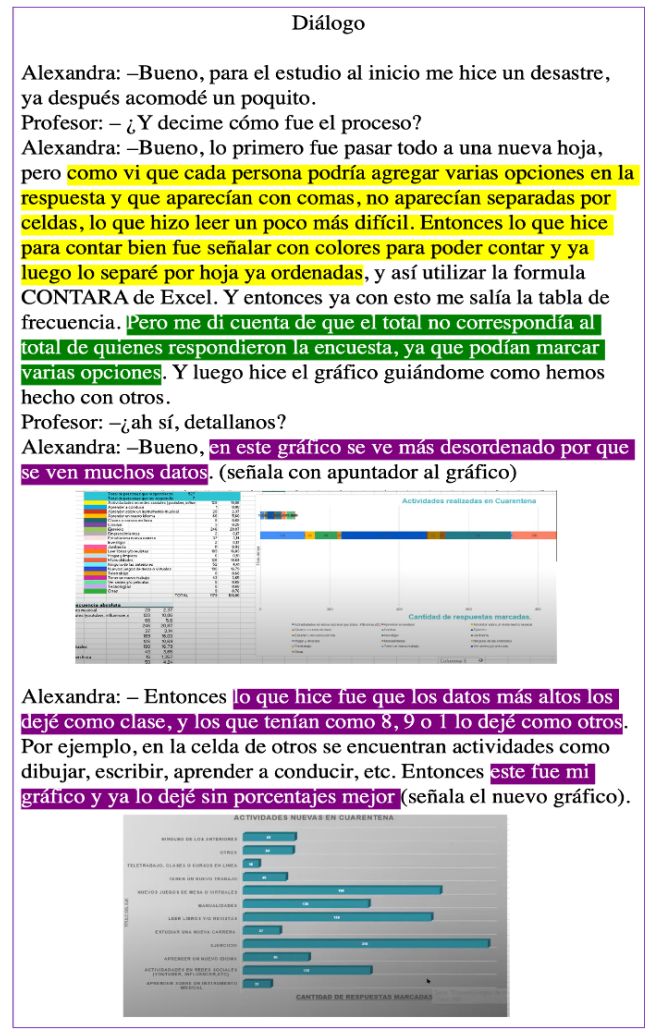

Organizar los datos (acción)

que lleva a la

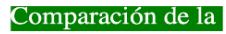

información (actividad),

Fuente: elaboración propia.

Para luego ofrecer una

Visualización de la

información (práctica)

Esta organización de prácticas (figura 8) permitió construir objetos como: moda, tablas de frecuencia absoluta y relativa, tipos de gráficos estadísticos y sus elementos. En esta problemática la media aritmética o la mediana no fueron medidas de tendencia central utilizadas para el análisis de las respuestas generadas en la encuesta, un estudio de estas hubiera quedado con un valor de uso artificial que no se pretende en una situación de aprendizaje. 
Figura 8. Organización de prácticas durante la exploración e interpretación de datos

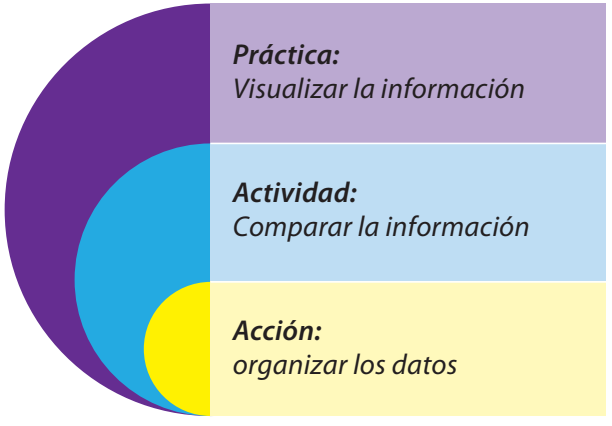

Fuente: elaboración propia.

Esta evolución permitió la exploración de diferentes gráficos estadísticos y a la vez tomar decisiones sobre lo informativo que puede llegar a ser o no este elemento gráfico (Figura 9). El estudiantado mostró seguridad al exponer los gráficos que habían construido, y, al mismo tiempo, afirmaron que no tuvieron que memorizar líneas para explicar y exponer la información; pues fue algo que vivieron y tuvieron la oportunidad de analizar.

Figura 9. Gráficos estadísticos creados por los estudiantes para el análisis y la comunicación de la información.

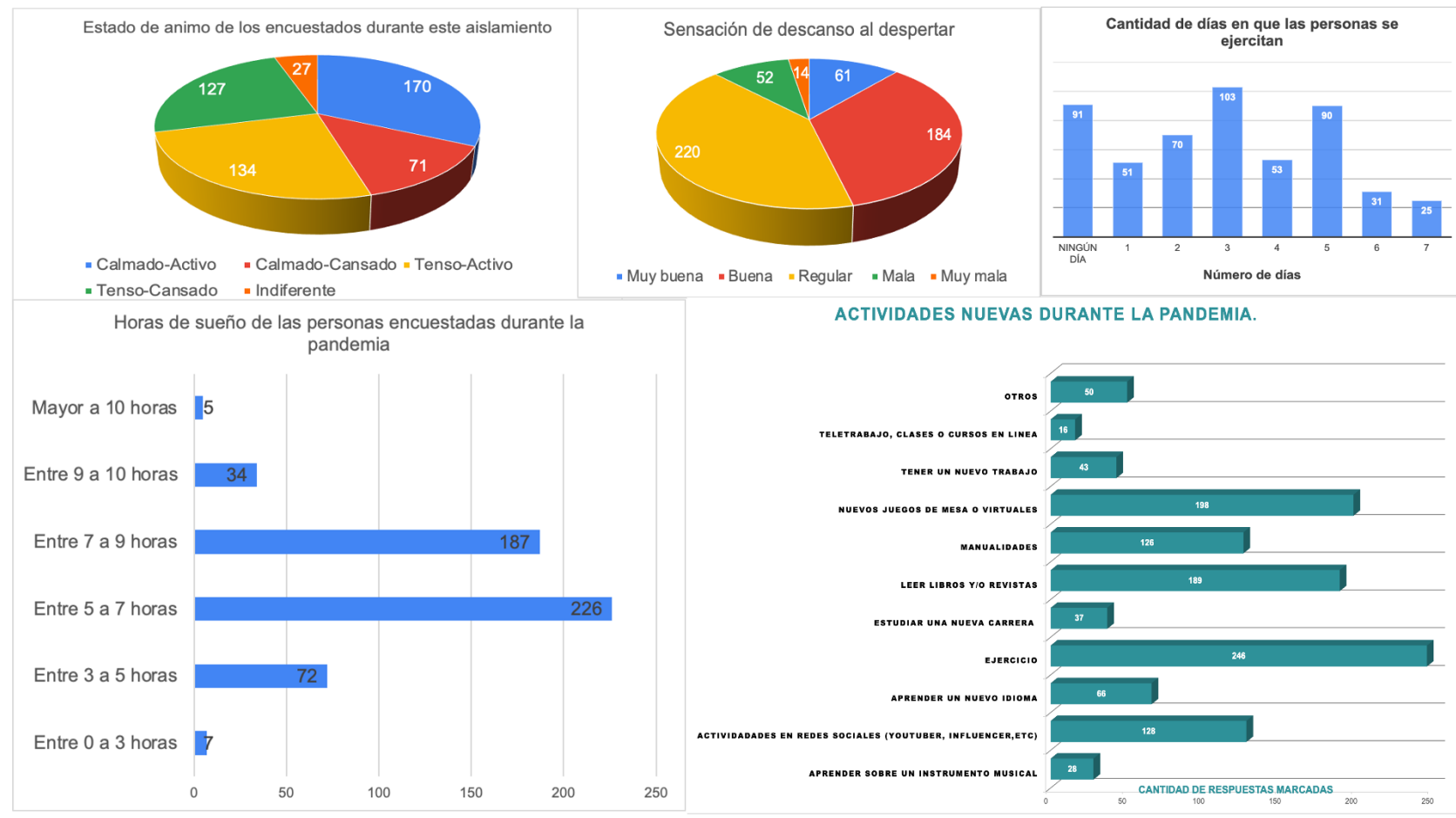

Fuente: elaboración propia. 


\section{SIINTESIS Y REFLEXIONES FINALES}

Con respecto al objetivo de este estudio, se logró diseñar, implementar y analizar el ciclo de investigación estadística ante un proyecto creado por un grupo de estudiantes de secundaria, quienes viven en diferentes localidades del país pero que compartieron una problemática en común. Pensar en el escenario de la educación estocástica contribuyó a tener en cuenta características para desarrollar propuestas que integran herramientas de estadística o probabilidad en el estudio de la información, construir inferencias y apreciar la incertidumbre.

Se reflexiona sobre la naturaleza epistemológica que tiene la didáctica de la estadística (campo de investigación) y educación estocástica (relacionado con el hacer del docente y la variedad de elementos complejos del sistema educativo) con respecto a las otras didácticas en matemática. Los trabajos en la línea de la educación estocástica no están tan centrados en el objeto en sí, sino en proyectos como lo es el ciclo PPDAC. Esto permite que el estudiante viva una constante situación de aprendizaje en el sentido socio-epistemológico, al estudiar una problemática real, pero sobre todo funcional en su contexto.

Teóricamente y con el apoyo del ciclo PPDAC, se evidencian las relaciones entre dos organizaciones de prácticas. La organización de prácticas presente en la recolección de datos influyó a nivel de acción a la estructura de prácticas presente para el análisis y la comunicación de la información, por el hecho de que los estudiantes ya habían construido el conocimiento de dato. Es importante evidenciar, en trabajos de investigación o académicos de corte socio-epistemológico, modelos dinámicos entre organizaciones de prácticas.

Durante la problematización del saber realizada desde la Teoría Socio-epistemológica, se reflexiona sobre la importancia de la práctica de inferencia asociada en el estudio de la estadística, combinatoria y probabilidad. La inferencia comprendida como la construcción de estimaciones sobre el fenómeno en un futuro y el contraste de hipótesis, aceptando la relatividad que tanto esas estimaciones como hipótesis pueden ser modificables. El tener en cuenta esta práctica ayudó a evidenciar en la implementación del ciclo a estructurar en forma intencional de prácticas descritas en los resultados.

Se concluye que, ante el estudio de fenómenos con comportamiento aleatorio, también existe la comparación de los datos y esto da la relación inmediata con el estudio del cambio y su naturaleza.

Para este caso en particular, reflexionar con el estudiantado sobre diferentes tamaños de muestra introdujo la importancia de valorar la representatividad de la muestra ante la población que se está estudiando. Se necesitan más trabajos en la línea de la educación estocástica y del Pensamiento y Lenguaje Variacional en la Socioepistemología, para continuar caracterizando la forma en cómo, a nivel de variabilidad, se piensa para analizar estos fenómenos de naturaleza no determinista.

Por otro lado, la escuela se norma o guía por lo propuesto en los programas curriculares, en su mayoría ofrece una evolución conceptual por temas: tipos de estadística, estudio de la muestra y población, tipos de variables, clasificación de esas variables, tablas de frecuencia (absoluta, relativa y relativa porcentual), medidas de tendencia central y gráficos estadísticos, entre otros. En los resultados se prueba que al centrarse en prácticas permite la construcción de objetos matemáticos, pero rompe con el orden de la evolución conceptual tradicional. Como prospectiva de investigación queda pendiente realizar trabajos comparativos para evidenciar el desempeño de los estudiantes formados con un enfoque en las prácticas ante pruebas estandarizadas que siguen estando basadas en el objeto.

Con respecto al rol docente, al realizar este tipo de proyectos o trabajar con fenómenos con comportamiento aleatorio hace que experimentemos ansiedad matemática, por el miedo a la incertidumbre. Es normal tener este sentimiento, lo importante es aceptarlo y aventurarse a estudiar problemas de naturaleza estocástica; pues en definitiva nutrirá al estudiantado en desarrollar un pensamiento estocástico 
que le ayudará a construir y usar herramientas para el análisis de este tipo de comportamientos que están presenten en problemas en la Ciencia sin resolver.

Por último, este tipo de experiencias didácticas nos acerca a conocer temas en otras disciplinas. Particularmente en este trabajo, se estudiaron en las clases formas de redactar o comunicarse, salud comunitaria, diseño publicitario para compartir la información, entre otras.

Estas propuestas podrían integrarse en los centros educativos para realizar proyectos colaborativos entre docentes de asignaturas y potenciar el análisis con la integración de materias que permitan vivir proyectos interdisciplinarios ante problemáticas que sean de interés en la formación del estudiante. Esto propicia a realizar una educación para el desarrollo sostenible de la sociedad.

\section{Agradecimientos}

Trabajo realizado en el ámbito del proyecto Comunidades de Aprendizaje Matemático (ED3569) del Departamento de Educación Matemática de la Escuela de Matemática de la Universidad de Costa Rica.

\section{REFERENCIAS}

Alsina, Á., Vásquez, C., Muñiz-Rodríguez, L., y Rodríguez-Muñiz, L. J. (2020). ¿Cómo promover la alfabetización estadística y probabilística en contexto? Estrategias y recursos a partir de la COVID-19 para Educación Primaria. Epsilon - Revista de Educación Matemática, (104):99-128.

Artigue, M. (2020). Didactic Engineering in Mathematics Education. In Encyclopedia of Mathematics Education. https://doi.org/10.1007/978-3-030-15789-0_44

Barboza Robles, Y. R. (2020). Competencia digital docente en el contexto de la Universidad Estatal a Distancia de Costa Rica. Innovaciones Educativas, 22(33):88-105. Retrieved from https://doi. org/10.22458/ie.v22i33.2954

Cantoral, R. (2019). Socioepistemology in Mathematics Education. In Encyclopedia of Mathematics Education. Retrieved from https://doi.org/10.1007/978-3-319-77487-9_100041-1

Cantoral, R., Montiel, G., y Reyes-Gasperini, D. (2015a). Análisis del discurso Matemático Escolar en los libros de texto, una mirada desde la Teoría Socioepistemológica. Avances de Investigación En Educación Matemática, (8):9-28. Retrieved from https://doi.org/10.35763/aiem.v1i8.123

Cantoral, R., Montiel, G., y Reyes-Gasperini, D. (2015b). El programa socioepistemológico de investigación en Matemática Educativa: el caso de Latinoamérica. Revista Latinoamericana de Investigación En Matemática Educativa, 18(1):5-17.

Carranza-Marchena, P., y Zamora-Sánchez, G. (2020). Desafíos y oportunidades en tiempos del COVID-19: contexto pedagógico desde la Universidad Nacional y la Universidad Estatal a Distancia. Innovaciones Educativas, 22(Especial):162 - 170. https://doi.org/10.22458/ie.v22iEspecial.3154

Huarcaya-Victoria J. (2020). Consideraciones sobre la salud mental en la pandemia de COVID-19. Revista Peruana de Medicina Experimental y Salud Publica, 37(2):327-334. http://dx.doi.org/10.17843/ rpmesp.2020.372.5419

Lopes, C. (2012). A Educação Estocástica na Infância. Revista Electrônica de Educação, 6(1):160-174.

Ministerio de Educación Pública. (2012). Programas de estudio en matemáticas. San José: MEP.

Nieuwenhuijsen, M. (9 de abril del 2020). ¿Cómo afecta la pandemia a la salud urbana?. The conversation. Recuperado de https://theconversation.com/

Ornell, F., Schuch, J.B., Sordi, A.O., y Kessler, F. (2020) "Pandemic fear" and COVID-19: mental health burden and strategies. Braz J Psychiatry, 42:232-235. http://dx.doi.org/10.1590/1516-4446-2020-0008 
Piaget, J. (1976). Piaget's Theory. In Piaget and His School. Retrieved from https://doi. org/10.1007/978-3-642-46323-5_2

Peralta, G., Carozzo, T., Sierra, M., y Bu, E. (2020). Enfermedad por coronavirus (COVID-19): la pandemia según la evidencia actual. Innovare: Revista De Ciencia y Tecnología, 9(1):15-27. https://doi. org/10.5377/innovare.v9i1.965

Ramírez-Ortiz, J., Castro-Quintero, D., Lerma-Córdoba, C., Yela-Ceballos, F., y Escobar-Córdoba, F. (2020). Consecuencias de la pandemia COVID-19 en la Salud Mental asociadas al aislamiento social. SciELO Preprints, 1-21. doi: https://doi.org/10.1590/SCIELOPREPRINTS.303

Vásquez Ortiz, C. (2020). Educación estocástica: una herramienta para formar ciudadanos de sostenibilidad. Matemáticas, Educación y Sociedad, 3(2):1-20.

Vega Jiménez, P. (2021). Costa Rica: dos pandemias en un siglo. Historia y Comunicación Social, 26(Especial):1-10. https://doi.org/10.5209/hics.74236

Vigotsky, L. (1986). Though and Language. MIT Press.

Wild, C., y Pfannkuch, M. (1999). Statistical Thinking in Empirical Enquiry. International Statistical Review, 67(3):223-265. 